\title{
Fourier series of sums of products of poly-Bernoulli functions and their applications
}

\author{
Taekyun Kimª, Dae San Kim ${ }^{b}$, Dmitry V. Dolgy ${ }^{\mathrm{c}}$, Jin-Woo Park ${ }^{\mathrm{d}, *}$ \\ ${ }^{a}$ Department of Mathematics, Kwangwoon University, Seoul 139-701, Republic of Korea. \\ ${ }^{b}$ Department of Mathematics, Sogang University, Seoul 121-742, Republic of Korea. \\ ${ }^{c}$ Hanrimwon, Kwangwoon University, Seoul 139-701, Republic of Korea. \\ ${ }^{d}$ Department of Mathematics Education, Daegu University, Gyeongsan-si, Gyeongsangbuk-do, 712-714, Republic of Korea.
}

Communicated by A. Meskhi

\begin{abstract}
In this paper, we consider three types of sums of products of poly-Bernoulli functions and derive Fourier series expansions of them. In addition, we express those three types of functions in terms of Bernoulli functions. (C)2017 All rights reserved.
\end{abstract}

Keywords: Fourier series, Bernoulli polynomial, poly-Bernoulli polynomial, poly-Bernoulli function.

2010 MSC: 11B68, 11B83, 42A16.

\section{Introduction}

As is well-known, the Bernoulli polynomials $\mathrm{B}_{\mathrm{m}}(\mathrm{x})$ are given by the generating function

$$
\frac{t}{e^{t}-1} e^{x t}=\sum_{m=0}^{\infty} B_{m}(x) \frac{t^{m}}{m !} .
$$

For any integer $r$, the poly-Bernoulli polynomials of index $r \mathbb{B}_{m}^{(r)}(x)$ are given by the generating function, (see $[1-3,7,11-15,17,20])$,

$$
\frac{L i_{r}\left(1-e^{-t}\right)}{e^{t}-1} e^{x t}=\sum_{m=0}^{\infty} \mathbb{B}_{m}^{(r)}(x) \frac{t^{m}}{m !},
$$

where $\operatorname{Li}_{r}(x)=\sum_{m=1}^{\infty} \frac{x^{m}}{m^{r}}$ is the $r$ th polylogarithmic function for $r \geqslant 1$ and a rational function for $r \leqslant 0$.

Note here that our definition of poly-Bernoulli polynomials is slightly different from the original definition of Kaneko's. Indeed, $\mathbb{B}_{m}^{(r)}(x)=\tilde{\mathbb{B}}_{m}^{(r)}(x-1)$, where $\tilde{\mathbb{B}}_{\mathfrak{m}}^{(r)}(x)$ denotes the Kaneko's poly-Bernoulli polynomials of index $r$, (see [7]). We also observe that

$$
\mathbb{B}_{\mathfrak{m}}^{(1)}(x)=B_{\mathfrak{m}}(x), \mathbb{B}_{0}^{(r)}(x)=1, \mathbb{B}_{\mathfrak{m}}^{(0)}(x)=x^{m}, \mathbb{B}_{m}^{(0)}=\delta_{\mathfrak{m}, 0}
$$

\footnotetext{
${ }^{*}$ Corresponding author

Email addresses: tkkim@kw.ac.kr (Taekyun Kim), dskim@sogang.ac.kr (Dae San Kim), dvdolgy@gmail.com (Dmitry V. Dolgy), a0417001@knu.ac.kr (Jin-Woo Park)

doi:10.22436/jnsa.010.05.10
} 
As $\mathbb{B}_{m}^{(r)}(x)$ is an Appell sequence,

$$
\frac{d}{d x} \mathbb{B}_{m}^{(r)}(x)=m B_{m-1}^{(r)}(x),(m \geqslant 1) .
$$

In addition,

$$
\frac{\mathrm{d}}{\mathrm{d} x}\left(\mathrm{Li}_{\mathrm{r}+1}(x)\right)=\frac{1}{x} \operatorname{Li} i_{\mathrm{r}}(x) .
$$

We claim that

$$
\mathbb{B}_{m}^{(r+1)}(1)-\mathbb{B}_{m}^{(r+1)}(0)=\mathbb{B}_{m-1}^{(r)}(0),(m \geqslant 1) .
$$

Indeed,

$$
\sum_{m=0}^{\infty}\left(\mathbb{B}_{\mathfrak{m}}^{(r+1)}(1)-\mathbb{B}_{m}^{(r+1)}(0)\right) \frac{t^{m}}{m !}=\operatorname{Li} i_{r+1}\left(1-e^{-t}\right),
$$

and differentiation on both sides with respect to $t$ gives

$$
\sum_{m=0}^{\infty}\left(\mathbb{B}_{m+1}^{(r+1)}(1)-\mathbb{B}_{m+1}^{(r+1)}(0)\right) \frac{t^{m}}{m !}=\frac{L i_{r}\left(1-e^{-t}\right)}{e^{t}-1}=\sum_{m=0}^{\infty} \mathbb{B}_{m}^{(r)}(0) \frac{t^{m}}{m !} .
$$

Recall that, for a polynomial $p(x) \in \mathbb{Q}[x]$ with $\operatorname{deg} p(x) \leqslant m$,

$$
p(x)=\sum_{j=0}^{m} a_{j} B_{j}(x), a_{j} \in \mathbb{Q},
$$

where (see [8-10])

$$
a_{0}=\int_{0}^{1} p(x) d x, a_{j}=\frac{1}{j !}\left(p^{(j-1)}(1)-p^{(j-1)}(0)\right), \text { for } j=1, \ldots, m .
$$

Now, we apply them to $p(x)=\mathbb{B}_{m}^{(r+1)}(x)$, and let

$$
\mathbb{B}_{m}^{(r+1)}(x)=\sum_{j=0}^{m} a_{j} B_{j}(x) .
$$

Then

$$
a_{0}=\int_{0}^{1} \mathbb{B}_{m}^{(r+1)}(x) d x=\frac{1}{m+1}\left(\mathbb{B}_{m+1}^{(r+1)}(1)-\mathbb{B}_{m+1}^{(r+1)}(0)\right)=\frac{1}{m+1} \mathbb{B}_{m}^{(r)}(0),
$$

and, for $1 \leqslant j \leqslant m$,

$$
a_{j}=\frac{(m)_{j-1}}{j !}\left(\mathbb{B}_{m-j+1}^{(r+1)}(1)-\mathbb{B}_{m-j+1}^{(r+1)}(0)\right)=\frac{1}{m+1}\left(\begin{array}{c}
m+1 \\
j
\end{array}\right) \mathbb{B}_{m-j}^{(r)}(0) .
$$

So

$$
\begin{aligned}
\mathbb{B}_{m}^{(r+1)}(x) & =\frac{1}{m+1} \mathbb{B}_{m}^{(r)}(0)+\frac{1}{m+1} \sum_{j=1}^{m}\left(\begin{array}{c}
m+1 \\
j
\end{array}\right) \mathbb{B}_{m-j}^{(r)}(0) B_{j}(x) \\
& =\frac{1}{m+1} \mathbb{B}_{m}^{(r)}(0)+\frac{1}{m+1} \sum_{j=0}^{m-1}\left(\begin{array}{c}
m+1 \\
j+1
\end{array}\right) \mathbb{B}_{j}^{(r)}(0) B_{m-j}(x) \\
& =\frac{1}{m+1} \sum_{j=0}^{m}\left(\begin{array}{c}
m+1 \\
j+1
\end{array}\right) \mathbb{B}_{j}^{(r)}(0) B_{m-j}(x) .
\end{aligned}
$$

This corresponds to Theorem 2.1 in [20]. Note that in that paper, the poly-Bernoulli polynomials are defined by yet another generating function. 
For any real number $x$, we let

$$
\langle x\rangle=x-\lfloor x\rfloor \in[0,1)
$$

denote the fractional part of $x$.

Fourier series expansion of higher-order Bernoulli functions were treated in the recent paper [16]. Here we consider the following three types of sums of products of poly-Bernoulli functions and derive Fourier series expansions of them. In addition, we express those three types of functions in terms of Bernoulli functions.

(1) $\alpha_{m}(\langle x\rangle)=\sum_{k=0}^{m} \mathbb{B}_{k}^{(r+1)}(\langle x\rangle) \mathbb{B}_{m-k}^{(s+1)}(\langle x\rangle),(m \geqslant 1)$;

(2) $\beta_{m}(\langle x\rangle)=\sum_{k=0}^{m} \frac{1}{k !(m-k) !} \mathbb{B}_{k}^{(r+1)}(\langle x\rangle) \mathbb{B}_{m-k}^{(s+1)}(\langle x\rangle),(m \geqslant 1)$;

(3) $\gamma_{m}(\langle x\rangle)=\sum_{k=1}^{m-1} \frac{1}{k(m-k)} \mathbb{B}_{k}^{(r+1)}(\langle x\rangle) \mathbb{B}_{m-k}^{(s+1)}(\langle x\rangle),(m \geqslant 2)$.

For elementary facts about Fourier analysis, the reader may refer to any book (for example, see [18, 21]). As to $\gamma_{m}(\langle x\rangle)$, we note that the following polynomial identity follows immediately from Theorems 4.1 and 4.2, which is in turn derived from the Fourier series expansion of $\gamma_{\mathrm{m}}(<x>)$ :

$$
\begin{aligned}
\sum_{k=1}^{m-1} \frac{1}{k(m-k)} \mathbb{B}_{k}^{(r+1)}(x) \mathbb{B}_{m-k}^{(s+1)}(x)= & \frac{1}{m}\left(\Lambda_{m+1}-\frac{1}{m(m+1)} \mathbb{B}_{m}^{(r)}-\frac{1}{m(m+1)} \mathbb{B}_{m}^{(s)}\right)+\frac{1}{m} \sum_{l=1}^{m}\left(\begin{array}{c}
m \\
l
\end{array}\right)\left(\Lambda_{m-l+1}\right. \\
& \left.+\frac{1}{m-l+1}\left(\mathbb{B}_{m-l}^{(r)}+\mathbb{B}_{m-l}^{(s)}\right)\left(H_{m-1}-H_{m-l}\right)\right) B_{l}(x),
\end{aligned}
$$

where $H_{l}=\sum_{j=1}^{l} \frac{1}{j}$ are the harmonic numbers and

$$
\Lambda_{l}=\sum_{k=1}^{l-1} \frac{1}{k(l-k)}\left(\mathbb{B}_{k}^{(r+1)} \mathbb{B}_{l-k-1}^{(s)}+\mathbb{B}_{k}^{(s+1)} \mathbb{B}_{l-k-1}^{(r)}+\mathbb{B}_{k-1}^{(r)} \mathbb{B}_{l-k-1}^{(s)}\right) .
$$

The obvious polynomial identities can be derived also for $\alpha_{m}(\langle x\rangle)$ and $\beta_{m}(\langle x\rangle)$ from Theorems 2.1 and 2.2, and Theorems 3.1 and 3.2, respectively. It is remarkable that from the Fourier series expansion of the function $\sum_{k=1}^{m-1} \frac{1}{k(m-k)} B_{k}(\langle x\rangle) B_{m-k}(\langle x\rangle)$ we can derive the Faber-Pandharipande-Zagier identity (see [5]) and the Miki's identity (see $[4,6,19])$.

\section{Sums of products of poly-Bernoulli functions of the first type}

For integers $r, s, m$ with $m \geqslant 1$, we let

$$
\begin{aligned}
\alpha_{m}(x) & =\sum_{k=0}^{m} \mathbb{B}_{k}^{(r+1)}(x) \mathbb{B}_{m-k}^{(s+1)}(x), \\
\alpha_{m}^{\prime}(x) & =\sum_{k=0}^{m}\left\{k \mathbb{B}_{k-1}^{(r+1)}(x) \mathbb{B}_{m-k}^{(s+1)}(x)+(m-k) \mathbb{B}_{k}^{(r+1)}(x) \mathbb{B}_{m-k-1}^{(s+1)}(x)\right\} \\
& =\sum_{k=1}^{m} k \mathbb{B}_{k-1}^{(r+1)}(x) \mathbb{B}_{m-k}^{(s+1)}(x)+\sum_{k=0}^{m-1}(m-k) \mathbb{B}_{k}^{(r+1)}(x) \mathbb{B}_{m-k-1}^{(s+1)}(x) \\
& =\sum_{k=0}^{m-1}(k+1) \mathbb{B}_{k}^{(r+1)}(x) \mathbb{B}_{m-1-k}^{(s+1)}(x)+\sum_{k=0}^{m-1}(m-k) \mathbb{B}_{k}^{(r+1)}(x) \mathbb{B}_{m-1-k}^{(s+1)}(x) \\
& =(m+1) \sum_{k=0}^{m-1} \mathbb{B}_{k}^{(r+1)}(x) \mathbb{B}_{m-1-k}^{(s+1)}(x)=(m+1) \alpha_{m-1}(x),(m \geqslant 1) .
\end{aligned}
$$

From this, we see that

$$
\int_{0}^{1} \alpha_{m}(x) d x=\frac{1}{m+2}\left(\alpha_{m+1}(1)-\alpha_{m+1}(0)\right) .
$$


We observe that

$$
\begin{aligned}
\alpha_{m}(1)-\alpha_{m}(0)= & \sum_{k=0}^{m}\left(\mathbb{B}_{k}^{(r+1)}(1) \mathbb{B}_{m-k}^{(s+1)}(1)-\mathbb{B}_{k}^{(r+1)} \mathbb{B}_{m-k}^{(s+1)}\right) \\
= & \sum_{k=1}^{m-1}\left(\mathbb{B}_{k}^{(r+1)}(1) \mathbb{B}_{m-k}^{(s+1)}(1)-\mathbb{B}_{k}^{(r+1)} \mathbb{B}_{m-k}^{(s+1)}\right) \\
& +\left(\mathbb{B}_{m}^{(s+1)}(1)-\mathbb{B}_{m}^{(s+1)}\right)+\left(\mathbb{B}_{m}^{(r+1)}(1)-\mathbb{B}_{m}^{(r+1)}\right) \\
= & \sum_{k=1}^{m-1}\left(\left(\mathbb{B}_{k}^{(r+1)}+\mathbb{B}_{k-1}^{(r)}\right)\left(\mathbb{B}_{m-k}^{(s+1)}+\mathbb{B}_{m-k-1}^{(s)}\right)-\mathbb{B}_{k}^{(r+1)} \mathbb{B}_{m-k}^{(s+1)}\right)+\mathbb{B}_{m-1}^{(s)}+\mathbb{B}_{m-1}^{(r)} \\
= & \sum_{k=1}^{m-1}\left(\mathbb{B}_{k}^{(r+1)} \mathbb{B}_{m-k-1}^{(s)}+\mathbb{B}_{k-1}^{(r)} \mathbb{B}_{m-k}^{(s+1)}+\mathbb{B}_{k-1}^{(r)} \mathbb{B}_{m-k-1}^{(s)}\right)+\mathbb{B}_{m-1}^{(s)}+\mathbb{B}_{m-1}^{(r)} \\
= & \sum_{k=0}^{m-1} \mathbb{B}_{k}^{(r+1)} \mathbb{B}_{m-k-1}^{(s)}+\sum_{k=0}^{m-1} \mathbb{B}_{k}^{(s+1)} \mathbb{B}_{m-k-1}^{(r)}+\sum_{k=1}^{m-1} \mathbb{B}_{k-1}^{(r)} \mathbb{B}_{m-k-1}^{(s)} .
\end{aligned}
$$

For $m \geqslant 1$, let $\triangle_{m}=\triangle_{m}(r, s)=\alpha_{m}(1)-\alpha_{m}(0)$. Then

$$
\alpha_{\mathrm{m}}(0)=\alpha_{\mathrm{m}}(1) \Longleftrightarrow \triangle_{\mathrm{m}}=0, \quad \int_{0}^{1} \alpha_{\mathrm{m}}(\mathrm{x}) \mathrm{d} x=\frac{1}{\mathrm{~m}+2} \triangle_{\mathrm{m}+1} .
$$

Now, we are going to consider the function

$$
\alpha_{m}(\langle x\rangle)=\sum_{k=0}^{m} \mathbb{B}_{k}^{(r+1)}(\langle x\rangle) \mathbb{B}_{m-k}^{(s+1)}(\langle x\rangle),(m \geqslant 1),
$$

defined on $(-\infty, \infty)$, which is periodic with period 1 .

The Fourier series of $\alpha_{m}(\langle x\rangle)$ is

$$
\sum_{n=-\infty}^{\infty} A_{n}^{(m)} e^{2 \pi i n x}
$$

where

$$
A_{n}^{(m)}=\int_{0}^{1} \alpha_{m}(\langle x\rangle) e^{-2 \pi i n x} d x=\int_{0}^{1} \alpha_{m}(x) e^{-2 \pi i n x} d x .
$$

We are now ready to determine the Fourier coefficients $A_{m}^{(m)}$.

Case $1: n \neq 0$.

$$
\begin{aligned}
A_{n}^{(m)} & =\int_{0}^{1} \alpha_{m}(x) e^{-2 \pi i n x} d x \\
& =-\frac{1}{2 \pi i n}\left[\alpha_{m}(x) e^{-2 \pi i n x}\right]_{0}^{1}+\frac{1}{2 \pi i n} \int_{0}^{1} \alpha_{m}^{\prime}(x) e^{-2 \pi i n x} d x \\
& =\frac{m+1}{2 \pi i n} A_{n}^{(m-1)}-\frac{1}{2 \pi i n} \triangle_{m} \\
& =\frac{m+1}{2 \pi i n}\left(\frac{m}{2 \pi i n} A_{n}^{(m-2)}-\frac{1}{2 \pi i n} \triangle_{m-1}\right)-\frac{1}{2 \pi i n} \triangle_{m} \\
& =\frac{(m+1) m}{(2 \pi i n)^{2}} A_{n}^{(m-2)}-\frac{m+1}{(2 \pi i n)^{2}} \triangle_{m-1}-\frac{1}{2 \pi i n} \triangle_{m}
\end{aligned}
$$




$$
\begin{aligned}
& =\frac{(m+1) m}{(2 \pi i n)^{2}}\left(\frac{m-1}{2 \pi i n} A_{n}^{(m-3)}-\frac{1}{2 \pi i n} \triangle_{m-2}\right)-\frac{m+1}{(2 \pi i n)^{2}} \triangle_{m-1}-\frac{1}{2 \pi i n} \triangle_{m} \\
& =\frac{(m+1)_{3}}{(2 \pi i n)^{3}} A_{n}^{(m-3)}-\sum_{j=1}^{3} \frac{(m+1)_{j-1}}{(2 \pi i n)^{j}} \triangle_{m-j+1} \\
& \vdots \\
& =\frac{(m+1) !}{(2 \pi i n)^{m}} A_{n}^{(0)}-\sum_{j=1}^{m} \frac{(m+1)_{j-1}}{(2 \pi i n)^{j}} \triangle_{m-j+1}
\end{aligned}
$$

where $A_{n}^{(0)}=\int_{0}^{1} e^{-2 \pi i n x} d x=0$. Thus

$$
A_{n}^{(m)}=-\frac{1}{m+2} \sum_{j=1}^{m} \frac{(m+2)_{j}}{(2 \pi i n)^{j}} \triangle_{m-j+1} .
$$

Case $2: n=0$.

$$
A_{0}^{(m)}=\int_{0}^{1} \alpha_{m}(x) d x=\frac{1}{m+2} \triangle_{m+1} .
$$

We recall the following facts about Bernoulli functions $B_{m}(\langle x\rangle)$ :

(a) for $m \geqslant 2$,

$$
B_{m}(\langle x\rangle)=-m ! \sum_{\substack{n=-\infty \\ n \neq 0}}^{\infty} \frac{e^{2 \pi i n x}}{(2 \pi i n)^{m}}
$$

(b) for $m=1$,

$$
-\sum_{\substack{n=-\infty \\ n \neq 0}}^{\infty} \frac{e^{2 \pi i n x}}{2 \pi i n}= \begin{cases}B_{1}(\langle x\rangle), & \text { for } x \notin \mathbb{Z}, \\ 0, & \text { for } x \in \mathbb{Z} .\end{cases}
$$

$\alpha(\langle x\rangle)(m \geqslant 1)$ is piecewise $C^{\infty}$. Moreover, $\alpha_{m}(\langle x\rangle)$ is continuous for those positive integers $m$ with $\triangle_{\mathfrak{m}}=0$ and discontinuous with jump discontinuities at integers for those positive integers with $\triangle_{\mathfrak{m}} \neq 0$.

Assume first that $m$ is a positive integer with $\triangle_{m}=0$. Then $\alpha_{m}(0)=\alpha_{m}(1) \cdot \alpha_{m}(\langle x\rangle)$ is piecewise $C^{\infty}$, and continuous. So the Fourier series of $\alpha_{m}(\langle x\rangle)$ converges uniformly to $\alpha_{m}(\langle x\rangle)$, and

$$
\begin{aligned}
& \alpha_{m}(\langle x\rangle)=\frac{1}{m+2} \triangle_{m+1}+\sum_{\substack{n=-\infty \\
n \neq 0}}^{\infty}\left(-\frac{1}{m+2} \sum_{j=1}^{m} \frac{(m+2)_{j}}{(2 \pi i n)^{j}} \triangle_{m-j+1}\right) e^{2 \pi i n x} \\
& =\frac{1}{m+2} \triangle_{m+1}+\frac{1}{m+2} \sum_{j=1}^{m}\left(\begin{array}{c}
m+2 \\
j
\end{array}\right) \triangle_{m-j+1}\left(-j ! \sum_{\substack{n=-\infty \\
n \neq 0}}^{\infty} \frac{e^{2 \pi i n x}}{(2 \pi i n)^{j}}\right) \\
& =\frac{1}{m+2} \triangle_{m+1}+\frac{1}{m+2} \sum_{j=2}^{m}\left(\begin{array}{c}
m+2 \\
j
\end{array}\right) \triangle_{m-j+1} B_{j}(\langle x\rangle)+\triangle_{m} \times \begin{cases}B_{1}(\langle x\rangle), & \text { for } x \notin \mathbb{Z}, \\
0, & \text { for } x \in \mathbb{Z} .\end{cases}
\end{aligned}
$$

Now, we can state our first theorem.

Theorem 2.1. For each positive integer $\mathrm{l}$, we let

$$
\triangle_{\mathrm{l}}=\triangle_{\mathrm{l}}(\mathrm{r}, \mathrm{s})=\sum_{\mathrm{k}=0}^{\mathrm{l}-1} \mathbb{B}_{\mathrm{k}}^{(\mathrm{r}+1)} \mathbb{B}_{\mathrm{l}-\mathrm{k}-1}^{(\mathrm{s})}+\sum_{\mathrm{k}=0}^{\mathrm{l}-1} \mathbb{B}_{\mathrm{k}}^{(\mathrm{s}+1)} \mathbb{B}_{\mathrm{l}-\mathrm{k}-1}^{(\mathrm{r})}+\sum_{\mathrm{k}=1}^{\mathrm{l}-1} \mathbb{B}_{\mathrm{k}-1}^{(\mathrm{r})} \mathbb{B}_{\mathrm{l}-\mathrm{k}-1}^{(\mathrm{s})} .
$$

Assume that $\triangle_{\mathrm{m}}=0$ for positive integer $\mathrm{m}$. Then we have the following. 
(a) $\sum_{k=0}^{m} \mathbb{B}_{k}^{(r+1)}(\langle x\rangle) \mathbb{B}_{m-k}^{(s+1)}(\langle x\rangle)$ has the Fourier series expansion

$$
\sum_{k=0}^{m} \mathbb{B}_{k}^{(r+1)}(\langle x\rangle) \mathbb{B}_{m-k}^{(s+1)}(\langle x\rangle)=\frac{1}{m+2} \triangle_{m+1}+\sum_{\substack{n=-\infty \\ n \neq 0}}^{\infty}\left(-\frac{1}{m+2} \sum_{j=1}^{m} \frac{(m+2)_{j}}{(2 \pi i n)^{j}} \triangle_{m-j+1}\right) e^{2 \pi i n x},
$$

for all $x \in(-\infty, \infty)$. Here the convergence is uniform.

(b)

$$
\sum_{k=0}^{m} \mathbb{B}_{k}^{(r+1)}(\langle x\rangle) \mathbb{B}_{m-k}^{(s+1)}(\langle x\rangle)=\frac{1}{m+2} \triangle_{m+1}+\frac{1}{m+2} \sum_{j=2}^{m}\left(\begin{array}{c}
m+2 \\
j
\end{array}\right) \triangle_{m-j+1} B_{j}(\langle x\rangle),
$$

for all $x \in(-\infty, \infty)$.

Here $\mathrm{B}_{j}(\langle x\rangle)$ is the Bernoulli function.

Assume next that $m$ is a positive integer with $\triangle_{\mathfrak{m}} \neq 0$. Then $\alpha_{\mathfrak{m}}(0) \neq \alpha_{m}(1)$. Then $\alpha_{m}(\langle x\rangle)$ is piecewise $C^{\infty}$, and discontinuous with jump discontinuities at integers. The Fourier series of $\alpha_{m}(\langle x\rangle)$ converges pointwise to $\alpha_{m}(\langle x\rangle)$, for $x \notin \mathbb{Z}$, and converges to

$$
\frac{1}{2}\left(\alpha_{m}(0)+\alpha_{m}(1)\right)=\alpha_{m}(0)+\frac{1}{2} \triangle_{m}=\sum_{k=0}^{m} \mathbb{B}_{k}^{(r+1)} \mathbb{B}_{m-k}^{(s+1)}+\frac{1}{2} \triangle_{m},
$$

for $x \in \mathbb{Z}$.

Now, we can state our second theorem.

Theorem 2.2. For each positive integer $l$, we let

$$
\triangle_{\mathrm{l}}=\triangle_{\mathrm{l}}(\mathrm{r}, \mathrm{s})=\sum_{\mathrm{k}=0}^{\mathrm{l}-1} \mathbb{B}_{\mathrm{k}}^{(\mathrm{r}+1)} \mathbb{B}_{\mathrm{l}-\mathrm{k}-1}^{(\mathrm{s})}+\sum_{\mathrm{k}=0}^{\mathrm{l}-1} \mathbb{B}_{\mathrm{k}}^{(\mathrm{s}+1)} \mathbb{B}_{\mathrm{l}-\mathrm{k}-1}^{(\mathrm{r})}+\sum_{\mathrm{k}=1}^{\mathrm{l}-1} \mathbb{B}_{\mathrm{k}-1}^{(\mathrm{r})} \mathbb{B}_{\mathrm{l}-\mathrm{k}-1}^{(\mathrm{s})} .
$$

Assume that $\triangle_{\mathrm{m}} \neq 0$, for a positive integer $\mathrm{m}$. Then we have the following.

(a)

$$
\begin{aligned}
\frac{1}{m+2} & \triangle_{m+1}+\sum_{\substack{n=-\infty \\
n \neq 0}}^{\infty}\left(-\frac{1}{m+2} \sum_{j=1}^{m} \frac{(m+2)_{j}}{(2 \pi i n)^{j}} \triangle_{m-j+1}\right) e^{2 \pi i n x} \\
& = \begin{cases}\sum_{k=0}^{m} \mathbb{B}_{k}^{(r+1)}(\langle x\rangle) \mathbb{B}_{m-k}^{(s+1)}(\langle x\rangle), & \text { for } x \notin \mathbb{Z}, \\
\sum_{k=0}^{m} \mathbb{B}_{k}^{(r+1)} \mathbb{B}_{m-k}^{(s+1)}+\frac{1}{2} \triangle_{m}, & \text { for } x \in \mathbb{Z} .\end{cases}
\end{aligned}
$$

(b)

Here the convergence is pointwise.

$$
\begin{aligned}
\frac{1}{m+2} \triangle_{m+1}+\frac{1}{m+2} \sum_{j=1}^{m}\left(\begin{array}{c}
m+2 \\
j
\end{array}\right) \triangle_{m-j+1} B_{j}(\langle x\rangle) & =\frac{1}{m+2} \sum_{j=0}^{m}\left(\begin{array}{c}
m+2 \\
j
\end{array}\right) \triangle_{m-j+1} B_{j}(\langle x\rangle) \\
& =\sum_{k=0}^{m} B_{k}^{(r+1)}(\langle x\rangle) B_{m-k}^{(s+1)}(\langle x\rangle), \text { for } x \notin \mathbb{Z}, \\
\frac{1}{m+2} \triangle_{m+1}+\frac{1}{m+2} \sum_{j=2}^{m}\left(\begin{array}{c}
m+2 \\
j
\end{array}\right) \triangle_{m-j+1} B_{j}(\langle x\rangle) & =\frac{1}{m+2} \sum_{\substack{j=0 \\
j \neq 1}}^{m}\left(\begin{array}{c}
m+2 \\
j
\end{array}\right) \triangle_{m-j+1} B_{j}(\langle x\rangle) \\
& =\sum_{k=0}^{m} \mathbb{B}_{k}^{(r+1)} \mathbb{B}_{m-k}^{(s+1)}+\frac{1}{2} \triangle_{m}, \text { for } x \in \mathbb{Z} .
\end{aligned}
$$

In the special case of $s=0$, we obtain the following results about the Fourier series of sums of products of poly-Bernoulli and Bernoulli functions. 
Theorem 2.3. For each positive integer $l \geqslant 2$, we let

$$
\tilde{\triangle}_{l}=\triangle_{l}(r, 0)=\mathbb{B}_{l-1}^{(r+1)}+\mathbb{B}_{l-2}^{(r)}+\sum_{k=0}^{l-1} B_{k} \mathbb{B}_{l-k-1}^{(r)}
$$

Assume that $\widetilde{\triangle}_{m}=0$ for an integer $m \geqslant 2$. Then we have the following.

(a) $\sum_{k=0}^{m} B_{k}^{(r+1)}(\langle x\rangle) B_{m-k}(\langle x\rangle)$ has the Fourier series expansion

$$
\sum_{k=0}^{m} \mathbb{B}_{k}^{(r+1)}(\langle x\rangle) B_{m-k}(\langle x\rangle)=\frac{1}{m+2} \tilde{\triangle}_{m+1}+\sum_{\substack{n=-\infty \\ n \neq 0}}^{\infty}\left(-\frac{1}{m+2} \sum_{j=1}^{m} \frac{(m+2)_{j}}{(2 \pi i n)^{j}} \tilde{\triangle}_{m-j+1}\right) e^{2 \pi i n x},
$$

for all $x \in(-\infty, \infty)$.

(b)

$$
\sum_{k=0}^{m} \mathbb{B}_{k}^{(r+1)}(\langle x\rangle) B_{m-k}(\langle x\rangle)=\frac{1}{m+2} \tilde{\triangle}_{m+1}+\frac{1}{m+2} \sum_{j=2}^{m}\left(\begin{array}{c}
m+2 \\
j
\end{array}\right) \tilde{\triangle}_{m-j+1} B_{j}(\langle x\rangle),
$$

for all $x \in(-\infty, \infty)$.

Theorem 2.4. For each positive integer $l \geqslant 2$, we let

$$
\widetilde{\triangle}_{l}=\triangle_{l}(r, 0)=\mathbb{B}_{l-1}^{(r+1)}+\mathbb{B}_{l-2}^{(r)}+\sum_{k=0}^{l-1} B_{k} \mathbb{B}_{l-k-1}^{(r)} .
$$

Assume that $\widetilde{\triangle}_{m} \neq 0$ for an integer $m \geqslant 2$. Then we have the following.

(a)

$$
\begin{aligned}
& \frac{1}{m+2} \tilde{\triangle}_{m+1}+\sum_{\substack{n=-\infty \\
n \neq 0}}^{\infty}\left(-\frac{1}{m+2} \sum_{j=1}^{m} \frac{(m+2)_{j}}{(2 \pi i n)^{j}} \tilde{\triangle}_{m-j+1}\right) e^{2 \pi i n x} \\
& \quad= \begin{cases}\sum_{k=0}^{m} \mathbb{B}_{k}^{(r+1)}(\langle x\rangle) B_{m-k}(\langle x\rangle), & \text { for } x \notin \mathbb{Z}, \\
\sum_{k=0}^{m} \mathbb{B}_{k}^{(r+1)} B_{m-k}+\frac{1}{2} \tilde{\triangle}_{m}, & \text { for } x \in \mathbb{Z} .\end{cases}
\end{aligned}
$$

(b)

$$
\begin{aligned}
& \frac{1}{m+2} \sum_{j=0}^{m}\left(\begin{array}{c}
m+2 \\
j
\end{array}\right) \tilde{\triangle}_{m-j+1} B_{j}(\langle x\rangle)=\sum_{k=0}^{m} \mathbb{B}_{k}^{(r+1)}(\langle x\rangle) B_{m-k}(\langle x\rangle), \text { for } x \notin \mathbb{Z}, \\
& \frac{1}{m+2} \sum_{\substack{j=0 \\
j \neq 1}}^{m}\left(\begin{array}{c}
m+2 \\
j
\end{array}\right) \tilde{\triangle}_{m-j+1} B_{j}(\langle x\rangle)=\sum_{k=0}^{m} B_{k}^{(r+1)} B_{m-k}+\frac{1}{2} \tilde{\triangle}_{m}, \text { for } x \in \mathbb{Z} .
\end{aligned}
$$

\section{Sums of products of poly-Bernoulli functions of the second type}

Let

$$
\beta_{m}(x)=\sum_{k=0}^{m} \frac{1}{k !(m-k) !} \mathbb{B}_{k}^{(r+1)}(x) \mathbb{B}_{m-k}^{(s+1)}(x),(m \geqslant 1),
$$




$$
\begin{aligned}
\beta_{m}^{\prime}(x) & =\sum_{k=0}^{m}\left\{\frac{k}{k !(m-k) !} \mathbb{B}_{k-1}^{(r+1)}(x) \mathbb{B}_{m-k}^{(s+1)}(x)+\frac{(m-k)}{k !(m-k) !} \mathbb{B}_{k}^{(r+1)}(x) \mathbb{B}_{m-k-1}^{(s+1)}(x)\right\} \\
& =\sum_{k=1}^{m} \frac{1}{(k-1) !(m-k) !} \mathbb{B}_{k-1}^{(r+1)}(x) \mathbb{B}_{m-k}^{(s+1)}(x)+\sum_{k=0}^{m-1} \frac{1}{k !(m-k-1) !} \mathbb{B}_{k}^{(r+1)} \mathbb{B}_{m-k-1}^{(s+1)}(x) \\
& =2 \sum_{k=0}^{m-1} \frac{1}{k !(m-1-k) !} \mathbb{B}_{k}^{(r+1)}(x) \mathbb{B}_{m-1-k}^{(s+1)}(x)=2 \beta_{m-1}(x) .
\end{aligned}
$$

From this we get $\left(\frac{\beta_{m+1}(x)}{2}\right)^{\prime}=\beta_{m}(x)$, and

$$
\int_{0}^{1} \beta_{\mathfrak{m}}(x) d x=\frac{1}{2}\left(\beta_{m+1}(1)-\beta_{\mathfrak{m}+1}(0)\right) .
$$

We observe that

$$
\begin{aligned}
\beta_{m}(1)-\beta_{m}(0)= & \sum_{k=0}^{m} \frac{1}{k !(m-k) !}\left(\mathbb{B}_{k}^{(r+1)}(1) \mathbb{B}_{m-k}^{(s+1)}(1)-\mathbb{B}_{k}^{(r+1)} \mathbb{B}_{m-k}^{(s+1)}\right) \\
= & \sum_{k=1}^{m-1} \frac{1}{k !(m-k) !}\left(\mathbb{B}_{k}^{(r+1)}(1) \mathbb{B}_{m-k}^{(s+1)}(1)-\mathbb{B}_{k}^{(r+1)} \mathbb{B}_{m-k}^{(s+1)}\right) \\
& +\frac{1}{m !}\left(\mathbb{B}_{m}^{(s+1)}(1)-\mathbb{B}_{m}^{(s+1)}\right)+\frac{1}{m !}\left(\mathbb{B}_{m}^{(r+1)}(1)-\mathbb{B}_{m}^{(r+1)}\right) \\
= & \sum_{k=1}^{m-1} \frac{1}{k !(m-k) !}\left(\left(\mathbb{B}_{k}^{(r+1)}+\mathbb{B}_{k-1}^{(r)}\right)\left(\mathbb{B}_{m-k}^{(s+1)}+\mathbb{B}_{m-k-1}^{(s)}\right)-\mathbb{B}_{k}^{(r+1)} \mathbb{B}_{m-k}^{(s+1)}\right) \\
& +\frac{1}{m !}\left(\mathbb{B}_{m}^{(s+1)}(1)-\mathbb{B}_{m}^{(s+1)}\right)+\frac{1}{m !}\left(\mathbb{B}_{m}^{(r+1)}(1)-\mathbb{B}_{m}^{(r+1)}\right) \\
= & \sum_{k=1}^{m-1} \frac{1}{k !(m-k) !}\left(\mathbb{B}_{k}^{(r+1)} \mathbb{B}_{m-k-1}^{(s)}+\mathbb{B}_{k-1}^{(r)} \mathbb{B}_{m-k}^{(s+1)}+\mathbb{B}_{k-1}^{(r)} \mathbb{B}_{m}^{(s)}-k-1\right)+\frac{1}{m !} \mathbb{B}_{m-1}^{(s)}+\frac{1}{m !} \mathbb{B}_{m-1}^{(r)} \\
= & \sum_{k=0}^{m-1} \frac{1}{k !(m-k) !} \mathbb{B}_{k}^{(r+1)} \mathbb{B}_{m-k-1}^{(s)}+\sum_{k=0}^{m-1} \frac{1}{k !(m-k) !} \mathbb{B}_{k}^{(s+1)} \mathbb{B}_{m}^{(r)}-k-1 \\
& +\sum_{k=1}^{m-1} \frac{1}{k !(m-k) !} \mathbb{B}_{k-1}^{(r)} \mathbb{B}_{m-k-1}^{(s)} \cdot
\end{aligned}
$$

For $m \geqslant 1$, let $\Omega_{m}=\Omega_{m}(r, s)=\beta_{m}(1)-\beta_{m}(0)$. Then

$$
\beta_{\mathrm{m}}(0)=\beta_{\mathrm{m}}(1) \Longleftrightarrow \Omega_{\mathrm{m}}=0 .
$$

In addition,

$$
\int_{0}^{1} \beta_{m}(x) d x=\frac{1}{2} \Omega_{m+1} .
$$

Now, we are going to consider the function

$$
\beta_{m}(\langle x\rangle)=\sum_{k=0}^{m} \frac{1}{k !(m-k) !} \mathbb{B}_{k}^{(r+1)}(\langle x\rangle) \mathbb{B}_{m-k}^{(s+1)}(\langle x\rangle),(m \geqslant 1),
$$

defined on $(-\infty, \infty)$, which is periodic with period 1 . 
The Fourier series of $\beta_{m}(\langle x\rangle)$ is

$$
\sum_{n=-\infty}^{\infty} B_{n}^{(m)} e^{2 \pi i n x}
$$

where

$$
\mathrm{B}_{\mathrm{n}}^{(\mathrm{m})}=\int_{0}^{1} \beta_{\mathrm{m}}(\langle x\rangle) e^{-2 \pi i n x} \mathrm{~d} x=\int_{0}^{1} \beta_{\mathrm{m}}(x) e^{-2 \pi i n x} \mathrm{~d} x
$$

Next, we want to determine the Fourier coefficients $B_{n}^{(m)}$.

Case $1: n \neq 0$.

$$
\begin{aligned}
B_{n}^{(m)} & =\int_{0}^{1} \beta_{m}(x) e^{-2 \pi i n x} d x \\
& =-\frac{1}{2 \pi i n}\left[\beta_{m}(x) e^{-2 \pi i n x}\right]+\frac{1}{2 \pi i n} \int_{0}^{1} \beta_{m}^{\prime}(x) e^{-2 \pi i n x} d x \\
& =-\frac{1}{2 \pi i n}\left(\beta_{m}(1)-\beta_{m}(0)\right)+\frac{2}{2 \pi i n} \int_{0}^{1} \beta_{m-1}(x) e^{-2 \pi i n x} d x \\
& =\frac{2}{2 \pi i n} B_{n}^{(m-1)}-\frac{1}{2 \pi i n} \Omega_{m} \\
& =\frac{2}{2 \pi i n}\left(\frac{2}{2 \pi i n} B_{n}^{(m-2)}-\frac{1}{2 \pi i n} \Omega_{m-1}\right)-\frac{1}{2 \pi i n} \Omega_{m} \\
& =\left(\frac{2}{2 \pi i n}\right)^{2} B_{n}^{(m-2)}-\sum_{j=1}^{2} \frac{2^{j-1}}{(2 \pi i n)^{j}} \Omega_{m-j-1} \\
& \vdots \\
& =\left(\frac{2}{2 \pi i n}\right)^{m} B_{n}^{(0)}-\sum_{j=1}^{m} \frac{2^{j-1}}{(2 \pi i n)^{j}} \Omega_{m-j+1} \\
& =-\sum_{j=1}^{m} \frac{2^{j-1}}{(2 \pi i n)^{j}} \Omega_{m-j=1}
\end{aligned}
$$

where $B_{n}^{(0)}=\int_{0}^{1} e^{-2 \pi i n x} d x=0$.

Case $2: n=0$.

$$
B_{0}^{(m)}=\int_{0}^{1} \beta_{m}(x) d x=\frac{1}{2} \Omega_{m+1} .
$$

$\beta_{m}(\langle x\rangle)(m \geqslant 1)$ is piecewise $C^{\infty}$. Moreover, $\beta_{m}(\langle x\rangle)$ is continuous for those positive integers with $\Omega_{\mathrm{m}}=0$, and discontinuous with jump discontinuities at integers for those positive integers with $\Omega_{\mathrm{m}} \neq 0$.

Assume first that $m$ is a positive integer with $\Omega_{\mathfrak{m}}=0$. Then $\beta_{\mathfrak{m}}(0)=\beta_{\mathfrak{m}}(1) . \beta_{\mathfrak{m}}(\langle x\rangle)$ is piecewise $C^{\infty}$, and continuous. Hence the Fourier series of $\beta_{m}(\langle x\rangle)$ converges uniformly to $\beta_{m}(\langle x\rangle)$, and

$$
\begin{aligned}
\beta_{m}(\langle x\rangle) & =\frac{1}{2} \Omega_{m+1}-\sum_{\substack{n=-\infty \\
n \neq 0}}^{\infty}\left(\sum_{j=1}^{m} \frac{2^{j-1}}{(2 \pi i n)^{j}} \Omega_{m-j+1}\right) e^{2 \pi i n x} \\
& =\frac{1}{2} \Omega_{m+1}+\sum_{j=1}^{m} \frac{2^{j-1}}{j !} \Omega_{m-j+1}\left(-j ! \sum_{\substack{n=-\infty \\
n \neq 0}}^{\infty} \frac{e^{2 \pi i n x}}{(2 \pi i n)^{j}}\right)
\end{aligned}
$$




$$
\begin{aligned}
& =\frac{1}{2} \Omega_{m+1}+\sum_{j=2}^{m} \frac{2^{j-1}}{j !} \Omega_{m-j+1} B_{j}(\langle x\rangle)+\Omega_{m} \times \begin{cases}B_{j}(\langle x\rangle), & \text { for } x \notin \mathbb{Z}, \\
0, & \text { for } x \in \mathbb{Z}\end{cases} \\
& =\sum_{\substack{j=0 \\
j \neq 1}}^{m} \frac{2^{j-1}}{j !} \Omega_{m-j+1} B_{j}(\langle x\rangle)+\Omega_{m} \times \begin{cases}B_{j}(\langle x\rangle), & \text { for } x \notin \mathbb{Z}, \\
0, & \text { for } x \in \mathbb{Z} .\end{cases}
\end{aligned}
$$

Now, we can state our first theorem.

Theorem 3.1. For each positive integer l, we let

$$
\Omega_{l}=\Omega_{l}(\mathrm{r}, \mathrm{s})=\sum_{\mathrm{k}=0}^{\mathrm{l}-1} \frac{1}{\mathrm{k} !(\mathrm{l}-\mathrm{k}) !} \mathbb{B}_{\mathrm{k}}^{(\mathrm{r}+1)} \mathbb{B}_{\mathrm{l}-\mathrm{k}-1}^{(\mathrm{s})}+\sum_{\mathrm{k}=0}^{\mathrm{l}-1} \frac{1}{\mathrm{k} !(\mathrm{l}-\mathrm{k}) !} \mathbb{B}_{\mathrm{k}}^{(\mathrm{s}+1)} \mathbb{B}_{\mathrm{l}-\mathrm{k}-1}^{(\mathrm{r})}+\sum_{\mathrm{k}=1}^{\mathrm{l}-1} \frac{1}{\mathrm{k} !(\mathrm{l}-\mathrm{k}) !} \mathbb{B}_{\mathrm{k}-1}^{(\mathrm{r})} \mathbb{B}_{\mathrm{l}-\mathrm{k}-1}^{(\mathrm{s})} .
$$

Assume that $\Omega_{\mathrm{m}}=0$ for a positive integer $\mathrm{m}$. Then we have the following.

(a) $\sum_{k=0}^{m} \frac{1}{k !(m-k) !} \mathbb{B}_{k}^{(r+1)}(\langle x\rangle) \mathbb{B}_{m-k}^{(s+1)}(\langle x\rangle)$ has the Fourier expansion

$$
\sum_{k=0}^{m} \frac{1}{k !(m-k) !} \mathbb{B}_{k}^{(r+1)}(\langle x\rangle) \mathbb{B}_{m-k}^{(s+1)}(\langle x\rangle)=\frac{1}{2} \Omega_{m+1}-\sum_{\substack{n=-\infty \\ n \neq 0}}^{\infty}\left(\sum_{j=1}^{m} \frac{2^{j-1}}{(2 \pi i n)^{j}} \Omega_{m-j+1}\right) e^{2 \pi i n x},
$$

for all $x \in(-\infty, \infty)$. Here the convergence is uniform.

(b)

$$
\sum_{k=0}^{m} \frac{1}{k !(m-k) !} \mathbb{B}_{k}^{(r+1)}(\langle x\rangle) \mathbb{B}_{m-k}^{(s+1)}(\langle x\rangle)=\sum_{\substack{j=0 \\ j \neq 1}}^{m} \frac{2^{j-1}}{j !} \Omega_{m-j+1} B_{j}(\langle x\rangle),
$$

for all $x \in(-\infty, \infty)$. Here $\mathrm{B}_{\mathrm{j}}(\langle x\rangle)$ is the Bernoulli function.

Assume next that $m$ is a positive integer with $\Omega_{m} \neq 0$. Then $\beta_{m}(0) \neq \beta_{m}(1) . \beta_{m}(\langle x\rangle)$ is piecewise $C^{\infty}$, and discontinuous with jump discontinuities at integers. Thus the Fourier series of $\beta_{m}(\langle x\rangle)$ converges pointwise to $\beta_{\mathrm{m}}(\langle x\rangle)$, for $x \notin \mathbb{Z}$, and converges to

$$
\frac{1}{2}\left(\beta_{m}(0)+\beta_{m}(1)\right)=\beta_{m}(0)+\frac{1}{2} \Omega_{m}=\sum_{k=0}^{m} \frac{1}{k !(m-k) !} \mathbb{B}_{k}^{(r+1)} \mathbb{B}_{m-k}^{(s+1)}+\frac{1}{2} \Omega_{m},
$$

for $x \in \mathbb{Z}$.

Next, we can state our second theorem.

Theorem 3.2. For each positive integer $\mathrm{l}$, we let

$$
\Omega_{l}=\Omega_{l}(\mathrm{r}, \mathrm{s})=\sum_{\mathrm{k}=0}^{\mathrm{l}-1} \frac{1}{\mathrm{k} !(\mathrm{l}-\mathrm{k}) !} \mathbb{B}_{\mathrm{k}}^{(\mathrm{r}+1)} \mathbb{B}_{\mathrm{l}-\mathrm{k}-1}^{(\mathrm{s})}+\sum_{\mathrm{k}=0}^{\mathrm{l}-1} \frac{1}{\mathrm{k} !(\mathrm{l}-\mathrm{k}) !} \mathbb{B}_{\mathrm{k}}^{(\mathrm{s}+1)} \mathbb{B}_{\mathrm{l}-\mathrm{k}-1}^{(\mathrm{r})}+\sum_{\mathrm{k}=1}^{\mathrm{l}-1} \frac{1}{\mathrm{k} !(\mathrm{l}-\mathrm{k}) !} \mathbb{B}_{\mathrm{k}-1}^{(\mathrm{r})} \mathbb{B}_{\mathrm{l}-\mathrm{k}-1}^{(\mathrm{s})} .
$$

Assume that $\Omega_{\mathrm{m}} \neq 0$ for a positive integer $\mathrm{m}$. Then we have the following.

(a)

$$
\begin{aligned}
& \frac{1}{2} \Omega_{m+1}-\sum_{\substack{n=-\infty \\
n \neq 0}}^{\infty}\left(\sum_{j=1}^{m} \frac{2^{j-1}}{(2 \pi i n)^{j}} \Omega_{m-j+1}\right) e^{2 \pi i n x} \\
& = \begin{cases}\sum_{k=0}^{m} \frac{1}{k !(m-k) !} \mathbb{B}_{k}^{(r+1)}(\langle x\rangle) \mathbb{B}_{m-k}^{(s+1)}(\langle x\rangle), & \text { for } x \notin \mathbb{Z}, \\
\sum_{k=0}^{m} \frac{1}{k !(m-k) !} \mathbb{B}_{k}^{(r+1)} \mathbb{B}_{m-k}^{(s+1)}+\frac{1}{2} \Omega_{m}, & \text { for } x \in \mathbb{Z},\end{cases}
\end{aligned}
$$

for all $x \in(-\infty, \infty)$. Here the convergence is pointwise. 
(b)

$$
\begin{aligned}
& \sum_{\substack{j=0 \\
m}}^{m} \frac{2^{j-1}}{j !} \Omega_{m-j+1} B_{j}(\langle x\rangle)=\sum_{k=0}^{m} \frac{1}{k !(m-k) !} \mathbb{B}_{k}^{(r+1)}(\langle x\rangle) \mathbb{B}_{m-k}^{(s+1)}(\langle x\rangle), \text { for } x \notin \mathbb{Z}, \\
& \sum_{\substack{j=0 \\
j \neq 1}}^{m} \frac{2^{j-1}}{j !} \Omega_{m-j+1} B_{j}(\langle x\rangle)=\sum_{k=0}^{m} \frac{1}{k !(m-k) !} \mathbb{B}_{k}^{(r+1)} \mathbb{B}_{m-k}^{(s+1)}+\frac{1}{2} \Omega_{m}, \text { for } x \in \mathbb{Z} .
\end{aligned}
$$

In the special case of $s=0$, we obtain the following results about the Fourier series of sums of products of poly-Bernoulli and Bernoulli functions.

Theorem 3.3. For each positive integer $l \geqslant 2$, we let

$$
\tilde{\Omega}_{l}=\Omega_{l}(r, 0)=\frac{1}{(l-1) !} \mathbb{B}_{l-1}^{(r+1)}+\frac{1}{(l-1) !} \mathbb{B}_{l-2}^{(r)}+\sum_{k=0}^{l-1} \frac{1}{k !(l-k) !} B_{k} \mathbb{B}_{l-k-1}^{(r)} .
$$

Assume that $\tilde{\Omega}_{\mathrm{m}}=0$ for an integer $\mathrm{m} \geqslant 2$. Then we have the following.

(a) $\sum_{k=0}^{m} \frac{1}{k !(m-k) !} \mathbb{B}_{k}^{(r+1)}(\langle x\rangle) B_{m-k}(\langle x\rangle)$ has the Fourier expansion

$$
\sum_{k=0}^{m} \frac{1}{k !(m-k) !} \mathbb{B}_{k}^{(r+1)}(\langle x\rangle) B_{m-k}(\langle x\rangle)=\frac{1}{2} \tilde{\Omega}_{m+1}-\sum_{\substack{n=-\infty \\ n \neq 0}}^{\infty}\left(\sum_{j=1}^{m} \frac{2^{j-1}}{(2 \pi i n)^{j}} \tilde{\Omega}_{m-j+1}\right) e^{2 \pi i n x},
$$

for all $x \in(-\infty, \infty)$.

(b)

$$
\sum_{k=0}^{m} \frac{1}{k !(m-k) !} B_{k}^{(r+1)}(\langle x\rangle) B_{m-k}(\langle x\rangle)=\sum_{\substack{j=0 \\ j \neq 1}}^{m} \frac{2^{j-1}}{j !} \tilde{\Omega}_{m-j+1} B_{j}(\langle x\rangle),
$$

for all $x \in(-\infty, \infty)$.

Theorem 3.4. For each positive integer $l \geqslant 2$, we let

$$
\tilde{\Omega}_{l}=\Omega_{l}(r, 0)=\frac{1}{(l-1) !} \mathbb{B}_{l-1}^{(r+1)}+\frac{1}{(l-1) !} \mathbb{B}_{l-2}^{(r)}+\sum_{k=0}^{l-1} \frac{1}{k !(l-k) !} B_{k} \mathbb{B}_{l-k-1}^{(r)} .
$$

Assume that $\tilde{\Omega}_{\mathrm{m}} \neq 0$ for a positive integer $\mathrm{m}$. Then we have the following.

(a)

$$
\begin{aligned}
& \frac{1}{2} \tilde{\Omega}_{m+1}-\sum_{\substack{n=-\infty \\
n \neq 0}}^{\infty}\left(\sum_{j=1}^{m} \frac{2^{j-1}}{(2 \pi i n)^{j}} \tilde{\Omega}_{m-j+1}\right) e^{2 \pi i n x} \\
& \quad=\left\{\begin{array}{lr}
\sum_{k=0}^{m} \frac{1}{k !(m-k) !} \mathbb{B}_{k}^{(r+1)}(\langle x\rangle) B_{m-k}(\langle x\rangle), & \text { for } x \notin \mathbb{Z}, \\
\sum_{k=0}^{m} \frac{1}{k !(m-k) !} \mathbb{B}_{k}^{(r+1)} B_{m-k}+\frac{1}{2} \tilde{\Omega}_{m}, & \text { for } x \in \mathbb{Z} .
\end{array}\right.
\end{aligned}
$$

(b)

$$
\begin{aligned}
& \sum_{j=0}^{m} \frac{2^{j-1}}{j !} \tilde{\Omega}_{m-j+1} B_{j}(\langle x\rangle)=\sum_{k=0}^{m} \frac{1}{k !(m-k) !} \mathbb{B}_{k}^{(r+1)}(\langle x\rangle) B_{m-k}(\langle x\rangle), \text { for } x \notin \mathbb{Z}, \\
& \sum_{\substack{j=0 \\
j \neq 1}}^{m} \frac{2^{j-1}}{j !} \tilde{\Omega}_{m-j+1} B_{j}(\langle x\rangle)=\sum_{k=0}^{m} \frac{1}{k !(m-k) !} \mathbb{B}_{k}^{(r+1)} B_{m-k}+\frac{1}{2} \tilde{\Omega}_{m}, \text { for } x \in \mathbb{Z} .
\end{aligned}
$$




\section{Sums of products of poly-Bernoulli functions of the third type}

Let

$$
\begin{aligned}
\gamma_{m}(x)= & \sum_{k=1}^{m-1} \frac{1}{k(m-k)} \mathbb{B}_{k}^{(r+1)}(x) \mathbb{B}_{m-k}^{(s+1)}(x),(m \geqslant 2), \\
\gamma_{m}^{\prime}(x)= & \sum_{k=1}^{m-1} \frac{1}{k(m-k)}\left\{k \mathbb{B}_{k-1}^{(r+1)}(x) \mathbb{B}_{m-k}^{(s+1)}(x)+(m-k) \mathbb{B}_{k}^{(r+1)}(x) \mathbb{B}_{m-k-1}^{(s+1)}(x)\right\} \\
= & \sum_{k=0}^{m-2} \frac{1}{m-1-k} \mathbb{B}_{k}^{(r+1)}(x) \mathbb{B}_{m-1-k}^{(s+1)}(x)+\sum_{k=1}^{m-1} \frac{1}{k} \mathbb{B}_{k}^{(r+1)}(x) \mathbb{B}_{m-1-k}^{(s+1)}(x) \\
= & \frac{1}{m-1} \mathbb{B}_{m-1}^{(s+1)}(x)+\sum_{k=1}^{m-2} \frac{1}{m-1-k} \mathbb{B}_{k}^{(r+1)}(x) \mathbb{B}_{m-1-k}^{(s+1)}(x) \\
& +\frac{1}{m-1} \mathbb{B}_{m-1}^{(r+1)}(x)+\sum_{k=1}^{m-2} \frac{1}{k} \mathbb{B}_{k}^{(r+1)}(x) \mathbb{B}_{m-1-k}^{(s+1)}(x) \\
= & \frac{1}{m-1} \mathbb{B}_{m-1}^{(r+1)}(x)+\frac{1}{m-1} \mathbb{B}_{m-1}^{(s+1)}(x)+(m-1) \sum_{k=1}^{m-2} \frac{1}{k(m-1-k)} \mathbb{B}_{k}^{(r+1)}(x) \mathbb{B}_{m-1}^{(s+1)}(x) \\
= & \frac{1}{m-1} \mathbb{B}_{m-1}^{(r+1)}(x)+\frac{1}{m-1} \mathbb{B}_{m-1}^{(s+1)}(x)+(m-1) \gamma_{m-1}(x) .
\end{aligned}
$$

So,

$$
\gamma_{m}^{\prime}(x)=\frac{1}{m-1}\left(\mathbb{B}_{m-1}^{(r+1)}(x)+\mathbb{B}_{m-1}^{(s+1)}(x)\right)+(m-1) \gamma_{m-1}(x) .
$$

From this, we have

$$
\begin{aligned}
&\left(\frac{1}{m}\left(\gamma_{m+1}(x)-\frac{1}{m(m+1)} \mathbb{B}_{m+1}^{(r+1)}(x)-\frac{1}{m(m+1)} \mathbb{B}_{m+1}^{(s+1)}(x)\right)\right)^{\prime}=\gamma_{m}(x) \\
& \int_{0}^{1} \gamma_{m}(x) d x= \frac{1}{m}\left[\gamma_{m+1}(x)-\frac{1}{m(m+1)} \mathbb{B}_{m+1}^{(r+1)}(x)-\frac{1}{m(m+1)} \mathbb{B}_{m+1}^{(s+1)}(x)\right]_{0}^{1} \\
&= \frac{1}{m}\left(\gamma_{m+1}(1)-\gamma_{m+1}(0)-\frac{1}{m(m+1)}\left(\mathbb{B}_{m+1}^{(r+1)}(1)-\mathbb{B}_{m+1}^{(r+1)}(0)\right)\right. \\
&\left.-\frac{1}{m(m+1)}\left(\mathbb{B}_{m+1}^{(s+1)}(1)-\mathbb{B}_{m+1}^{(s+1)}(0)\right)\right) \\
&= \frac{1}{m}\left(\gamma_{m+1}(1)-\gamma_{m+1}(0)-\frac{1}{m(m+1)} \mathbb{B}_{m}^{(r)}-\frac{1}{m(m+1)} \mathbb{B}_{m}^{(s)}\right)
\end{aligned}
$$

and

$$
\begin{aligned}
\gamma_{m}(1)-\gamma_{m}(0) & =\sum_{k=1}^{m-1} \frac{1}{k(m-k)}\left(\mathbb{B}_{k}^{(r+1)}(1) \mathbb{B}_{m-k}^{(s+1)}(1)-\mathbb{B}_{k}^{(r+1)} \mathbb{B}_{m-k}^{(s+1)}\right) \\
& =\sum_{k=1}^{m-1} \frac{1}{k(m-k)}\left(\left(\mathbb{B}_{k}^{(r+1)}+\mathbb{B}_{k-1}^{(r)}\right)\left(\mathbb{B}_{m-k}^{(s+1)}+\mathbb{B}_{m-k-1}^{(s)}\right)-\mathbb{B}_{k}^{(r+1)} \mathbb{B}_{m-k}^{(s+1)}\right) \\
& =\sum_{k=1}^{m-1} \frac{1}{k(m-k)}\left(\mathbb{B}_{k}^{(r+1)} \mathbb{B}_{m-k-1}^{(s)}+\mathbb{B}_{k-1}^{(r)} \mathbb{B}_{m-k}^{(s+1)}+\mathbb{B}_{k-1}^{(r)} \mathbb{B}_{m-k-1}^{(s)}\right)
\end{aligned}
$$


For $m \geqslant 2$, we let

$$
\Lambda_{m}=\Lambda_{m}(r, s)=\gamma_{m}(1)-\gamma_{m}(0)=\sum_{k=1}^{m-1} \frac{1}{k(m-k)}\left(\mathbb{B}_{k}^{(r+1)} \mathbb{B}_{m-k-1}^{(s)}+\mathbb{B}_{k}^{(s+1)} \mathbb{B}_{m-k-1}^{(r)}+\mathbb{B}_{k-1}^{(r)} \mathbb{B}_{m-k-1}^{(s)}\right) .
$$

Then $\gamma_{m}(0)=\gamma_{m}(1)$ if and only if $\Lambda_{m}=0$. Also,

$$
\int_{0}^{1} \gamma_{m}(x) d x=\frac{1}{m}\left(\Lambda_{m+1}-\frac{1}{m(m+1)} \mathbb{B}_{m}^{(r)}-\frac{1}{m(m+1)} \mathbb{B}_{m}^{(s)}\right) .
$$

We are now going to consider

$$
\gamma_{m}(\langle x\rangle)=\sum_{k=1}^{m-1} \frac{1}{k(m-k)} \mathbb{B}_{k}^{(r+1)}(\langle x\rangle) \mathbb{B}_{m-k}^{(s+1)}(\langle x\rangle),(m \geqslant 2),
$$

defined on $(-\infty, \infty)$, which is periodic with period 1 .

The Fourier series of $\gamma_{m}(\langle x\rangle)$ is

$$
\sum_{n=-\infty}^{\infty} C_{n}^{(m)} e^{2 \pi i n x}
$$

where

$$
C_{n}^{(m)}=\int_{0}^{1} \gamma_{m}(\langle x\rangle) e^{-2 \pi i n x} d x=\int_{0}^{1} \gamma_{m}(x) e^{-2 \pi i n x} d x
$$

We are now ready to determine the Fourier coefficients $C_{n}^{(m)}$.

Case $1: n \neq 0$.

We can show that

$$
\int_{0}^{1} \mathbb{B}_{l}^{(r+1)}(x) e^{-2 \pi i n x} d x=-\sum_{k=1}^{l} \frac{(l)_{k-1}}{(2 \pi i n)^{k}} \mathbb{B}_{l-k^{\prime}}^{(r)}
$$

and we let

$$
\begin{aligned}
\Phi_{m, r}= & \sum_{k=1}^{m-1} \frac{(m-1)_{k-1}}{(2 \pi i n)^{k}} \mathbb{B}_{m-k-1}^{(r)} \\
C_{n}^{(m)}= & -\frac{1}{2 \pi i n}\left[\gamma_{m}(x) e^{-2 \pi i n x}\right]_{0}^{1}+\frac{1}{2 \pi i n} \int_{0}^{1} \gamma_{m}^{\prime}(x) e^{-2 \pi i n x} \mathrm{~d} x \\
= & -\frac{1}{2 \pi i n}\left(\gamma_{m}(1)-\gamma_{m}(0)\right) \\
& +\frac{1}{2 \pi i n} \int_{0}^{1}\left((m-1) \gamma_{m-1}(x)+\frac{1}{m-1} \mathbb{B}_{m-1}^{(r+1)}(x)+\frac{1}{m-1} \mathbb{B}_{m-1}^{(s+1)}(x)\right) e^{-2 \pi i n x} d x \\
= & -\frac{1}{2 \pi i n} \Lambda_{m}+\frac{m-1}{2 \pi i n} C_{n}^{(m-1)}+\frac{1}{2 \pi i n(m-1)} \int_{0}^{1} \mathbb{B}_{m-1}^{(r+1)}(x) e^{-2 \pi i n x} d x \\
& +\frac{1}{2 \pi i n(m-1)} \int_{0}^{1} \mathbb{B}_{m-1}^{(s+1)}(x) e^{-2 \pi i n x} d x \\
= & -\frac{1}{2 \pi i n} \Lambda_{m}+\frac{m-1}{2 \pi i n} C_{n}^{(m-1)}-\frac{1}{2 \pi i n(m-1)} \sum_{k=1}^{m-1} \frac{(m-1)_{k-1}}{(2 \pi i n)^{k}} \mathbb{B}_{m-k-1}^{(r)} \\
& -\frac{1}{2 \pi i n(m-1)} \sum_{k=1}^{m-1} \frac{(m-1)_{k-1}}{(2 \pi i n)^{k}} \mathbb{B}_{m-k-1}^{(s)}
\end{aligned}
$$




$$
\begin{aligned}
= & \frac{m-1}{2 \pi i n} C_{n}^{(m-1)}-\frac{1}{2 \pi i n} \Lambda_{m}-\frac{1}{2 \pi i n(m-1)} \Phi_{m, r}-\frac{1}{2 \pi i n(m-1)} \Phi_{m, s} \\
= & \frac{m-1}{2 \pi i n}\left(\frac{m-2}{2 \pi i n} C_{n}^{(m-2)}-\frac{1}{2 \pi i n} \Lambda_{m-1}-\frac{1}{2 \pi i n(m-2)} \Phi_{m-1, r}\right. \\
& \left.-\frac{1}{2 \pi i n(m-2)} \Phi_{m-1, s}\right)-\frac{1}{2 \pi i n} \Lambda_{m}-\frac{1}{2 \pi i n(m-1)} \Phi_{m, r}-\frac{1}{2 \pi i n(m-1)} \Phi_{m, s} \\
= & \frac{(m-1)_{2}}{(2 \pi i n)^{2}} C_{n}^{(m-2)}-\sum_{j=1}^{2} \frac{(m-1)_{j-1}}{(2 \pi i n)^{j}} \Lambda_{m-j+1}-\sum_{j=1}^{2} \frac{(m-1)_{j-1}}{(2 \pi i n)^{j}(m-j)} \Phi_{m-j+1, r} \\
& -\sum_{j=1}^{2} \frac{(m-1)_{j-1}}{(2 \pi i n)^{j}(m-j)} \Phi_{m-j+1, s} \\
\vdots & -\sum_{j=1}^{m-1} \frac{(m-1)_{j-1}}{(2 \pi i n)^{j}} \Lambda_{m-j+1}-\sum_{j=1}^{m-1} \frac{(m-1)_{j-1}}{(2 \pi i n)^{j}(m-j)} \Phi_{m-j+1, r}-\sum_{j=1}^{m-1} \frac{(m-1)_{j-1}}{(2 \pi i n)^{j}(m-j)} \Phi_{m-j+1, s}
\end{aligned}
$$

where we note that $C_{n}^{(1)}=0$. We observe here that

$$
\begin{aligned}
\sum_{j=1}^{m-1} \frac{(m-1)_{j-1}}{(2 \pi i n)^{j}(m-j)} \Phi_{m-j+1, r} & =\sum_{j=1}^{m-1} \sum_{k=1}^{m-j} \frac{(m-1)_{j+k-2}}{(2 \pi i n)^{j+k}(m-j)} \mathbb{B}_{m-j-k}^{(r)} \\
& =\frac{1}{m} \sum_{l=1}^{m} \frac{(m)_{l}}{(2 \pi i n)^{l}} \frac{\mathbb{B}_{m-l}^{(r)}}{m-l+1}\left(H_{m-1}-H_{m-l}\right) .
\end{aligned}
$$

Thus,

$$
C_{n}^{(m)}=-\frac{1}{m} \sum_{l=1}^{m} \frac{(m)_{l}}{(2 \pi i n)^{l}}\left\{\Lambda_{m-l+1}+\frac{1}{m-l+1}\left(\mathbb{B}_{m-l}^{(r)}+\mathbb{B}_{m-l}^{(s)}\right)\left(H_{m-1}-H_{m-l}\right)\right\} .
$$

Case $2: n=0$.

$$
C_{0}^{(m)}=\int_{0}^{1} \gamma_{m}(x) d x=\frac{1}{m}\left(\Lambda_{m+1}-\frac{1}{m(m+1)} \mathbb{B}_{m}^{(r)}-\frac{1}{m(m+1)} \mathbb{B}_{m}^{(s)}\right)
$$

$\gamma_{m}(\langle x\rangle)(m \geqslant 2)$ is piecewise $C^{\infty}$. Moreover, $\gamma_{m}(\langle x\rangle)$ is continuous for those integers $m \geqslant 2$ with $\Lambda_{m}=0$, and discontinuous with jump discontinuities at integers for those integers $m \geqslant 2$ with $\Lambda_{m} \neq 0$.

Assume first that $m$ is an integer $\geqslant 2$ with $\Lambda_{m}=0$. Then $\gamma_{m}(0)=\gamma_{m}(1) \cdot \gamma_{m}(\langle x\rangle)$ is piecewise $C^{\infty}$, and continuous. So the Fourier series of $\gamma_{m}(\langle x\rangle)$ converges uniformly to $\gamma_{m}(\langle x\rangle)$, and

$$
\begin{aligned}
\gamma_{m}(\langle x\rangle)= & \frac{1}{m}\left(\Lambda_{m+1}-\frac{1}{m(m+1)} \mathbb{B}_{m}^{(r)}-\frac{1}{m(m+1)} \mathbb{B}_{m}^{(s)}\right)-\frac{1}{m} \sum_{\substack{n=-\infty \\
n \neq 0}}^{\infty}\left\{\sum _ { l = 0 } ^ { m } \frac { ( m ) _ { l } } { ( 2 \pi i n ) ^ { l } } \left(\Lambda_{m-l+1}\right.\right. \\
& \left.\left.+\frac{1}{m-l+1}\left(\mathbb{B}_{m-l}^{(r)}+\mathbb{B}_{m-l}^{(s)}\right)\left(H_{m-1}-H_{m-l}\right)\right)\right\} e^{2 \pi i n x} \\
= & \frac{1}{m}\left(\Lambda_{m+1}-\frac{1}{m(m+1)} \mathbb{B}_{m}^{(r)}-\frac{1}{m(m+1)} \mathbb{B}_{m}^{(s)}\right) \\
& +\frac{1}{m} \sum_{\substack{l=1 \\
m}}^{m}\left(\begin{array}{c}
m \\
l
\end{array}\right)\left(\Lambda_{m-l+1}+\frac{1}{m-l+1}\left(\mathbb{B}_{m-l}^{(r)}+\mathbb{B}_{m-l}^{(s)}\right)\left(H_{m-1}-H_{m-l}\right)\right) \\
& \times\left(-l \sum_{\substack{n=-\infty \\
n \neq 0}}^{-l(2 \pi i n)^{l}}\right)
\end{aligned}
$$




$$
\begin{aligned}
= & \frac{1}{m}\left(\Lambda_{m+1}-\frac{1}{m(m+1)} \mathbb{B}_{m}^{(r)}-\frac{1}{m(m+1)} \mathbb{B}_{m}^{(s)}\right) \\
& +\frac{1}{m} \sum_{l=2}^{m}\left(\begin{array}{c}
m \\
l
\end{array}\right)\left(\Lambda_{m-l+1}+\frac{1}{m-l+1}\left(\mathbb{B}_{m-l}^{(r)}+\mathbb{B}_{m-l}^{(s)}\right)\left(H_{m-1}-H_{m-l}\right)\right) B_{l}(\langle x\rangle) \\
& +\Lambda_{m} \times \begin{cases}B_{1}(\langle x\rangle), & \text { for } x \notin \mathbb{Z}, \\
0, & \text { for } x \in \mathbb{Z} .\end{cases}
\end{aligned}
$$

Now, we can state our first theorem.

Theorem 4.1. For each positive integer $l \geqslant 2$, we let

$$
\Lambda_{l}=\Lambda_{l}(\mathrm{r}, \mathrm{s})=\sum_{\mathrm{k}=1}^{\mathrm{l}-1} \frac{1}{\mathrm{k}(\mathrm{l}-\mathrm{k})}\left(\mathbb{B}_{\mathrm{k}}^{(\mathrm{r}+1)} \mathbb{B}_{\mathrm{l}-\mathrm{k}-1}^{(\mathrm{s})}+\mathbb{B}_{\mathrm{k}}^{(\mathrm{s}+1)} \mathbb{B}_{\mathrm{l}-\mathrm{k}-1}^{(\mathrm{r})}+\mathbb{B}_{\mathrm{k}-1}^{(\mathrm{r})} \mathbb{B}_{\mathrm{l}-\mathrm{k}-1}^{(\mathrm{s})}\right)
$$

with $\Lambda_{1}=0$. Assume that $\Lambda_{\mathrm{m}}=0$ for a positive integer $\mathrm{m} \geqslant 2$. Then we have the following.

(a)

$$
\begin{aligned}
& \sum_{k=1}^{m-1} \frac{1}{k(m-k)} \mathbb{B}_{k}^{(r+1)}(\langle x\rangle) \mathbb{B}_{m-k}^{(s+1)}(\langle x\rangle) \\
& \quad=\frac{1}{m}\left(\Lambda_{m+1}-\frac{1}{m(m+1)} \mathbb{B}_{m}^{(r)}-\frac{1}{m(m+1)} \mathbb{B}_{m}^{(s)}\right) \\
& \quad-\frac{1}{m} \sum_{\substack{n=-\infty \\
n \neq 0}}^{\infty}\left\{\sum_{l=1}^{m} \frac{(m)_{l}}{(2 \pi i n)^{l}}\left(\Lambda_{m-l+1}+\frac{1}{m-l+1}\left(\mathbb{B}_{m-l}^{(r)}+\mathbb{B}_{m-l}^{(s)}\right)\left(H_{m-1}-H_{m-l}\right)\right)\right\} e^{2 \pi i n x},
\end{aligned}
$$

for all $x \in(-\infty, \infty)$. Here the convergence is uniform.

(b)

$$
\begin{aligned}
& \sum_{k=1}^{m-1} \frac{1}{k(m-k)} \mathbb{B}_{k}^{(r+1)}(\langle x\rangle) \mathbb{B}_{m-k}^{(s+1)}(\langle x\rangle) \\
& \quad=\frac{1}{m}\left(\Lambda_{m+1}-\frac{1}{m(m+1)} \mathbb{B}_{m}^{(r)}-\frac{1}{m(m+1)} \mathbb{B}_{m}^{(s)}\right) \\
& \quad+\frac{1}{m} \sum_{l=2}^{m}\left(\begin{array}{c}
m \\
l
\end{array}\right)\left(\Lambda_{m-l+1}+\frac{1}{m-l+1}\left(\mathbb{B}_{m-l}^{(r)}+\mathbb{B}_{m-l}^{(s)}\right)\left(H_{m-1}-H_{m-l}\right)\right) B_{l}(\langle x\rangle),
\end{aligned}
$$

for $\mathrm{x} \in(-\infty, \infty)$. Here $\mathrm{B}_{\mathrm{l}}(\langle\mathrm{x}\rangle)$ is the Bernoulli function.

Assume next that $m$ is an integer $\geqslant 2$ with $\Lambda_{m} \neq 0$. Then $\gamma_{m}(0) \neq \gamma_{m}(1) . \gamma_{m}(\langle x\rangle)$ is piecewise $\mathrm{C}^{\infty}$, and discontinuous with jump discontinuities at integers. So the Fourier series of $\gamma_{\mathrm{m}}(\langle x\rangle)$ converges pointwise to $\gamma_{\mathrm{m}}(\langle x\rangle)$, for $x \notin \mathbb{Z}$, and converges to

$$
\frac{1}{2}\left(\gamma_{m}(0)+\gamma_{m}(1)\right)=\gamma_{m}(0)+\frac{1}{2} \Lambda_{m}=\sum_{k=1}^{m-1} \frac{1}{k(m-k)} \mathbb{B}_{k}^{(r+1)} \mathbb{B}_{m-k}^{(s+1)}+\frac{1}{2} \Lambda_{m},
$$

for $x \in \mathbb{Z}$. Now, we can state our second theorem.

Theorem 4.2. For each positive integer $l \geqslant 2$, we let

$$
\Lambda_{\mathrm{l}}=\Lambda_{\mathrm{l}}(\mathrm{r}, \mathrm{s})=\sum_{\mathrm{k}=1}^{\mathrm{l}-1} \frac{1}{\mathrm{k}(\mathrm{l}-\mathrm{k})}\left(\mathbb{B}_{\mathrm{k}}^{(\mathrm{r}+1)} \mathbb{B}_{\mathrm{l}-\mathrm{k}-1}^{(\mathrm{s})}+\mathbb{B}_{\mathrm{k}}^{(\mathrm{s}+1)} \mathbb{B}_{\mathrm{l}-\mathrm{k}-1}^{(\mathrm{r})}+\mathbb{B}_{\mathrm{k}-1}^{(\mathrm{r})} \mathbb{B}_{\mathrm{l}-\mathrm{k}-1}^{(\mathrm{s})}\right),
$$

with $\Lambda_{1}=0$. Assume that $\Lambda_{\mathrm{m}} \neq 0$, for a positive integer $\mathrm{m} \geqslant 2$. Then we have the following. 
(a)

$$
\begin{aligned}
& \frac{1}{m}\left(\Lambda_{m+1}-\frac{1}{m(m+1)} \mathbb{B}_{m}^{(r)}-\frac{1}{m(m+1)} \mathbb{B}_{m}^{(s)}\right) \\
& -\frac{1}{m} \sum_{\substack{n=-\infty \\
n \neq 0}}^{\infty}\left\{\sum_{l=1}^{m} \frac{(m)_{l}}{(2 \pi i n)^{l}}\left(\Lambda_{m-l+1}+\frac{1}{m-l+1}\left(\mathbb{B}_{m-l}^{(r)}+\mathbb{B}_{m-l}^{(s)}\right)\left(H_{m-1}-H_{m-l}\right)\right)\right\} e^{2 \pi i n x} \\
& = \begin{cases}\sum_{k=1}^{m-1} \frac{1}{k(m-k)} \mathbb{B}_{k}^{(r+1)}(\langle x\rangle) \mathbb{B}_{m-k}^{(s+1)}(\langle x\rangle), & \text { for } x \notin \mathbb{Z}, \\
\sum_{k=1}^{m-1} \frac{1}{k(m-k)} \mathbb{B}_{k}^{(r+1)} \mathbb{B}_{m-k}^{(s+1)}+\frac{1}{2} \Lambda_{m}, & \text { for } x \in \mathbb{Z} .\end{cases}
\end{aligned}
$$

Here the convergence is pointwise.

(b)

$$
\begin{aligned}
& \frac{1}{m}\left(\Lambda_{m+1}-\frac{1}{m(m+1)} \mathbb{B}_{m}^{(r)}-\frac{1}{m(m+1)} \mathbb{B}_{m}^{(s)}\right)+\frac{1}{m} \sum_{l=1}^{m}\left(\begin{array}{c}
m \\
l
\end{array}\right)\left(\Lambda_{m-l+1}\right. \\
& \left.\quad+\frac{1}{m-l+1}\left(\mathbb{B}_{m-l}^{(r)}+\mathbb{B}_{m-l}^{(s)}\right)\left(H_{m-1}-H_{m-l}\right)\right) B_{l}(\langle x\rangle) \\
& =\sum_{k=1}^{m-1} \frac{1}{k(m-k)} \mathbb{B}_{k}^{(r+1)}(\langle x\rangle) \mathbb{B}_{m-k}^{(s+1)}(\langle x\rangle), \text { for } x \notin \mathbb{Z}, \\
& \frac{1}{m}\left(\Lambda_{m+1}-\frac{1}{m(m+1)} \mathbb{B}_{m}^{(r)}-\frac{1}{m(m+1)}+\mathbb{B}_{m}^{(s)}\right)+\frac{1}{m} \sum_{l=2}^{m}\left(\begin{array}{c}
m \\
l
\end{array}\right)\left(\Lambda_{m-l+1}\right. \\
& \left.\quad+\frac{1}{m-l+1}\left(\mathbb{B}_{m-l}^{(r)}+\mathbb{B}_{m-l}^{(s)}\right)\left(H_{m-1}-H_{m-l}\right)\right) B_{l}(\langle x\rangle) \\
& =\sum_{k=1}^{m-1} \frac{1}{k(m-k)} \mathbb{B}_{k}^{(r+1)} \mathbb{B}_{m-k}^{(s+1)}+\frac{1}{2} \Lambda_{m} .
\end{aligned}
$$

In the special case of $s=0$, we obtain the following results about the Fourier series of sums of products of poly-Bernoulli and Bernoulli functions.

Theorem 4.3. For each positive integer $l \geqslant 2$, we let

$$
\tilde{\Lambda}_{l}=\Lambda_{l}(\mathrm{r}, 0)=\frac{1}{l-1} \mathbb{B}_{l-1}^{(\mathrm{r}+1)}+\frac{1}{\mathrm{l}-1} \mathbb{B}_{l-2}^{(\mathrm{r})}+\sum_{\mathrm{k}=1}^{\mathrm{l}-1} \frac{1}{\mathrm{k}(\mathrm{l}-\mathrm{k})} \mathrm{B}_{\mathrm{k}} \mathbb{B}_{\mathrm{l-k}-1}^{(\mathrm{r})}
$$

with $\tilde{\Lambda}_{1}=0$. Assume that $\tilde{\Lambda}_{m}=0$ for a positive integer $m \geqslant 2$. Then we have the following.

(a)

$$
\begin{aligned}
& \sum_{k=1}^{m-1} \frac{1}{k(m-k)} \mathbb{B}_{k}^{(r+1)}(\langle x\rangle) B_{m-k}(\langle x\rangle) \\
& =\frac{1}{m}\left(\tilde{\Lambda}_{m+1}-\frac{1}{m(m+1)} \mathbb{B}_{m}^{(r)}\right)-\frac{1}{m} \sum_{\substack{n=-\infty \\
n \neq 0}}^{\infty}\left\{\sum _ { l = 1 } ^ { m - 1 } \frac { ( m ) _ { l } } { ( 2 \pi i n ) ^ { l } } \left(\tilde{\Lambda}_{m-l+1}\right.\right. \\
& \left.\left.\quad+\frac{\mathbb{B}_{m-l}^{(r)}}{m-l+1}\left(H_{m-1}-H_{m-l}\right)\right)+\frac{2 m !}{(2 \pi i n)^{m}} H_{m-1}\right\} e^{2 \pi i n x},
\end{aligned}
$$

for all $x \in(-\infty, \infty)$. 
(b)

$$
\begin{aligned}
& \sum_{k=1}^{m-1} \frac{1}{k(m-k)} \mathbb{B}_{k}^{(r+1)}(\langle x\rangle) B_{m-k}(\langle x\rangle) \\
& =\frac{1}{m}\left(\tilde{\Lambda}_{m+1}-\frac{1}{m(m+1)} \mathbb{B}_{k}^{(r)}\right)+\frac{1}{m} \sum_{l=2}^{m-1}\left(\begin{array}{c}
m \\
l
\end{array}\right)\left(\tilde{\Lambda}_{m-l+1}\right. \\
& \left.\quad+\frac{1}{m-l+1} \mathbb{B}_{m-l}^{(r)}\left(H_{m-1}-H_{m-l}\right)\right) B_{l}(\langle x\rangle)+\frac{2}{m} H_{m-1} B_{m}(\langle x\rangle)
\end{aligned}
$$

for all $x \in(-\infty, \infty)$.

Theorem 4.4. For each positive integer $l \geqslant 2$, let

$$
\tilde{\Lambda}_{l}=\Lambda_{l}(r, 0)=\frac{1}{l-1} \mathbb{B}_{l-1}^{(r+1)}+\frac{1}{l-1} \mathbb{B}_{l-2}^{(r)}+\sum_{k=1}^{l-1} \frac{1}{k(l-k)} B_{k} \mathbb{B}_{l-k-1}^{(r)}
$$

with $\tilde{\Lambda}_{1} \neq 0$. Assume that $\tilde{\Lambda}_{m} \neq 0$ for a positive integer $m \geqslant 2$. Then we have the following.

(a)

$$
\begin{aligned}
\frac{1}{m}\left(\tilde{\Lambda}_{m+1}-\frac{1}{m(m+1)} \mathbb{B}_{m}^{(r)}\right)-\frac{1}{m} \sum_{\substack{n=-\infty \\
n \neq 0}}^{\infty}\left\{\sum _ { l = 1 } ^ { m - 1 } \frac { ( m ) _ { l } } { ( 2 \pi i n ) ^ { l } } \left(\tilde{\Lambda}_{m-l+1}\right.\right. \\
\left.\left.+\frac{B_{m-l}^{(r)}}{m-l+1}\left(H_{m-1}-H_{m-l}\right)\right)+\frac{2 m !}{(2 \pi i n)^{m}} H_{m-1}\right\} e^{2 \pi i n x} \\
= \begin{cases}\sum_{k=1}^{m-1} \frac{1}{k(m-k)} \mathbb{B}_{k}^{(r+1)}(\langle x\rangle) B_{m-k}(\langle x\rangle), & \text { for } x \notin \mathbb{Z}, \\
\sum_{k=1}^{m-1} \frac{1}{k(m-k)} \mathbb{B}_{k}^{(r+1)} B_{m-k}+\frac{1}{2} \tilde{\Lambda}_{m}, & \text { for } x \in \mathbb{Z} .\end{cases}
\end{aligned}
$$

(b)

$$
\begin{aligned}
\frac{1}{m}\left(\Lambda_{m+1}-\frac{1}{m(m+1)} B_{m}^{(r)}\right)+\frac{1}{m} \sum_{l=1}^{m-1}\left(\begin{array}{c}
m \\
l
\end{array}\right)\left(\tilde{\Lambda}_{m-l+1}\right. \\
\left.\quad+\frac{1}{m-l+1} B_{m-l}^{(r)}\left(H_{m-1}-H_{m-l}\right)\right) B_{l}(\langle x\rangle)+\frac{2}{m} H_{m-1} B_{m}(\langle x\rangle) \\
=\sum_{k=1}^{m-1} \frac{1}{k(m-k)} B_{k}^{(r+1)}(\langle x\rangle) B_{m-k}(\langle x\rangle), \text { for } x \notin \mathbb{Z}, \\
\frac{1}{m}\left(\tilde{\Lambda}_{m+1}-\frac{1}{m(m+1)} \mathbb{B}_{m}^{(r)}\right)+\frac{1}{m} \sum_{l=2}^{m}\left(\begin{array}{c}
m \\
l
\end{array}\right)\left(\tilde{\Lambda}_{m-l+1}\right. \\
\left.\quad+\frac{1}{m-l+1} B_{m-l}^{(r)}\left(H_{m-1}-H_{m-l}\right)\right) B_{l}(\langle x\rangle)+\frac{2}{m} H_{m-1} B_{m}(\langle x\rangle) \\
=\sum_{k=1}^{m-1} \frac{1}{k(m-k)} \mathbb{B}_{k}^{(r+1)} B_{m-k}+\frac{1}{2} \tilde{\Lambda}_{m}, \text { for } x \in \mathbb{Z} .
\end{aligned}
$$

\section{Acknowledgment}

This research was supported by the Daegu University Research Grant, 2016. 


\section{References}

[1] T. Arakawa, M. Kaneko, On poly-Bernoulli numbers, Comment. Math. Univ. St. Paul., 48 (1999), 159-167. 1

[2] A. Bayad, Y. Hamahata, Multiple polylogarithms and multi-poly-Bernoulli polynomials, Funct. Approx. Comment. Math., 46 (2012), 45-61.

[3] D. V. Dolgy, D. S. Kim, T. Kim, T. Mansour, Degenerate poly-Bernoulli polynomials of the second kind, J. Comput. Anal. Appl., 21 (2016), 954-966. 1

[4] G. V. Dunne, C. Schubert, Bernoulli number identities from quantum field theory and topological string theory, Commun. Number Theory Phys., 7 (2013), 225-249. 1

[5] C. Faber, R. Pandharipande, Hodge integrals and Gromov-Witten theory, Invent. Math., 139 (2000), 173-199. 1

[6] I. M. Gessel, On Miki's identity for Bernoulli numbers, J. Number Theory, 110 (2005), 75-82. 1

[7] M. Kaneko, Poly-Bernoulli numbers, J. Théor. Nombres Bordeaux, 9 (1997), 221-228. 1

[8] D. S. Kim, D. V. Dolgy, T. Kim, S.-H. Rim, Some formulae for the product of two Bernoulli and Euler polynomials, Abstr. Appl. Anal., 2012 (2012), 15 pages. 1

[9] D. S. Kim, T. Kim, Bernoulli basis and the product of several Bernoulli polynomials, Int. J. Math. Math. Sci., 2012 (2012), 12 pages.

[10] D. S. Kim, T. Kim, Some identities of higher order Euler polynomials arising from Euler basis, Integral Transforms Spec. Funct., 24 (2013), 734-738. 1

[11] D. S. Kim, T. Kim, A note on degenerate poly-Bernoulli numbers and polynomials, Adv. Difference Equ., 2015 (2015), 8 pages. 1

[12] D. S. Kim, T. Kim, A note on poly-Bernoulli and higher-order poly-Bernoulli polynomials, Russ. J. Math. Phys., 22 (2015), 26-33.

[13] D. S. Kim, T. Kim, Higher-order Bernoulli and poly-Bernoulli mixed type polynomials, Georgian Math. J., 22 (2015), 265-272.

[14] D. S. Kim, T. Kim, H. I. Kwon, T. Mansour, Degenerate poly-Bernoulli polynomials with umbral calculus viewpoint, J. Inequal. Appl., 2015 (2015), 13 pages.

[15] D. S. Kim, T. Kim, T. Mansour, J.-J. Seo, Fully degenerate poly-Bernoulli polynomials with a q parameter, Filomat, 30 (2016), 1029-1035. 1

[16] T. Kim, D. S. Kim, S.-H. Rim, D. V. Dolgy, Fourier series of higher-order Bernoulli functions and their applications, J. Inequal. Appl., 2017 (2017), 7 pages. 1

[17] T. Kim, D. S. Kim, J.-J. Seo, Fully degenerate poly-Bernoulli numbers and polynomials, Open Math., 14 (2016), 545-556. 1

[18] J. E. Marsden, Elementary classical analysis, With the assistance of Michael Buchner, Amy Erickson, Adam Hausknecht, Dennis Heifetz, Janet Macrae and William Wilson, and with contributions by Paul Chernoff, István Fáry and Robert Gulliver, W. H. Freeman and Co., San Francisco, (1974). 1

[19] K. Shiratani, S. Yokoyama, An application of p-adic convolutions, Mem. Fac. Sci. Kyushu Univ. Ser. A, 36 (1982), 73-83. 1

[20] P. T. Young, Bernoulli and poly-Bernoulli polynomial convolutions and identities of p-adic Arakawa-Kaneko zeta functions, J. Number Theory, 12 (2016), 1295-1309. 1

[21] D. G. Zill, M. R. Cullen, Advanced engineering mathematics, second edition, Jones \& Bartlett Learning, Massachusetts, (2000). 1 